\title{
Distributed Power Control in Wireless Ad Hoc Networks Using Message Passing: Throughput Optimality and Network Utility Maximization
}

\author{
Aneesh Reddy, Sanjay Shakkottai, and Lei Ying
}

\begin{abstract}
This paper presents an algorithm for distributed power control and scheduling over wireless ad hoc-networks, where the data rate on each link depends on the transmission power levels at interfering links (non-convex coupling between link data rates). In this paper, we first consider a $K$-hop interference model. We describe a message passing algorithm that finds an optimal power allocation (schedule) in the case of line networks with a time complexity (in number of nodes $N$ ) that grows as $N$ for line networks. Further, we show that this algorithm, when combined with appropriate congestion-control and routing algorithms results in throughput-optimality and utility maximization over wireless networks. We further study a complete physical interference model, where our algorithms provide $\epsilon$-optimal solutions. Our results can also be extended to grid networks.
\end{abstract}

\section{INTRODUCTION}

In this paper, we study the problem of distributed power control in the context of utility maximization and throughput optimality over a wireless ad hoc network. This problem has received increasing attention over the recent past [1], [2], [3], [4], [5] where attention has been focused on developing joint congestion control, routing and scheduling algorithms using a (stochastic) network utility maximization framework.

Typically, the approach consists of formulating the network resource allocation problem as a convex optimization problem (by approximating the wireless physical layer [6]), and cross-layer solutions either are based on primal-dual algorithms for convex optimization [5], [7] and/or by means of a per-time-slot scheduling combined with a queue length based back-pressure algorithm [8], [9], [2], [10].

In either case, it is now understood that a key difficulty is in the distributed scheduling aspect (either for utility maximization or for queue stability). In wireless networks, the transmission rate of each link is dependent on the transmission decision (schedule) at other nodes as well as their actual transmit power levels (for transmitting nodes). This dependency between the capacity of links and transmission schedule is typically non-convex. Thus solving the scheduling problem at each time slot is difficult and acts as a bottle neck for the cross-layer optimization. A popular approach [5] is to suitably approximate the physical layer model in order to render it convex (e.g., [5] uses a high-SINR approximation

This work was partially supported by NSF Grants CNS-0347400, CNS-0519535, and CNS-0721380, the Darpa ITMANET program, and DTRA grant HDTRA1-08-1-0016. A. Reddy and S. Shakkottai are with the Department of Electrical and Computer Engineering, The University of Texas at Austin, and part of the Wireless Communications and Networking Group (WNCG). L. Ying is with the Department of Electrical and Computer Engineering, Iowa State University e-mail: \{aneesh, shakkott\}@mail.utexas.edu, leiying@iastate.edu. and appropriate variable transformations to formulate the power-control/scheduling problem as a geometric program).

In this paper, we take a different approach where we do not approximate the physical layer interactions across nodes. Instead, we use a message-passing approach [11] in order to to solve the non-convex scheduling/power-control problem in a distributed fashion with polynomial complexity. Messagepassing (MP) algorithms have been shown to be very successful in many communication contexts including iterative decoding of codes [12], and more recently in scheduling over graphs [13].

In this paper, our main contributions are as follows:

(i) For a $K$-hop interference model over a line network (nodes interact with up to $K$-hop neighbors), we describe a message-passing algorithm that exactly solves the distributed scheduling problem. Further, we show that that this algorithm when combined with an appropriate back-pressure scheme/congestion control mechanism leads to network throughput-optimality/utilitymaximization.

(ii) For a complete physical interference model (the pathloss exponent has a polynomial decay with appropriate degree with respect to distance between transmitter and receiver), we show that the message passing algorithm can be adapted to determine an $\epsilon$-optimal power allocation schedule.

\section{System Model: Line NeTWORK With $K$-HOP INTERFERENCE}

In this section, we describe the basic system model in the context of a line network with $K$-hop interference and use this model to develop a message-passing algorithm. Later, in section VII, we will show that this algorithm can be used to derive an $\epsilon$-optimal (defined in section VII) power control schedule with a complete physical interference model as well.

Network Model: Consider a wireless network represented by a directed graph $\mathcal{G}=(\mathcal{V}, \mathcal{E})$, where $\mathcal{V}=\{1,2,3, \ldots, N\}$ is the set of wireless nodes and $\mathcal{E}$ is the set of directed links. Denote by $(t, r)$ a directed link such that node $t$ is the transmitter and node $r$ is the receiver. In this paper, we focus on line networks as in Figure 1. The results, however can be extended to grid network and will be discussed in section III.

Note that $(t, r)$ is a link in a line network if node $t$ and $r$ are adjacent nodes, i.e., $|t-r|=1$. Given a link $l$, we let $t(l)$ denote the sender of the link, and $r(l)$ denote the 


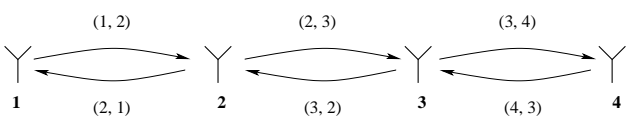

Fig. 1. Line Network

receiver. Two links are said to be adjacent if they share a common node, i.e., $l_{1}$ and $l_{2}$ are adjacent links if

$$
\left\{t\left(l_{1}\right), r\left(l_{1}\right)\right\} \cap\left\{t\left(l_{2}\right), r\left(l_{2}\right)\right\} \neq \emptyset .
$$

Let $\mathcal{A}(l)$ denote the set of adjacent links of link $l$, for example, $\mathcal{A}((1,2))=\{(2,1),(2,3),(3,2)\}$.

Discrete Power Level: We assume that each transmitter can transmit at one of $L$ different power levels. Define $\mathcal{P}$ to be the set of powers, so $\mathcal{P}=\left\{0, P_{1}, P_{2}, \ldots ., P_{L-1}\right\}$, where $P_{i}<P_{i+1}$. For ease of exposition, we assume that all transmitters have the identical power set (we note that it is straightforward to extend to the case where each transmitter has distinct power sets).

Channel Model: Let $\mathrm{X}$ be a $2 N-2$ vector such that $X_{l} \in$ $\mathcal{P}$ denotes the power at which transmitter $t(l)$ transmits. We assume a Gaussian channel model such that the rate attainable on link $l, R_{l}(\mathbf{X})$, is given by

$$
R_{l}(\mathbf{X})=\log \left(1+\operatorname{SINR}_{l}(\mathbf{X})\right)
$$

where

$$
\operatorname{SINR}_{l}(\mathbf{X})=\frac{G_{l l} X_{l}}{N_{l}+\sum_{h \in \mathcal{E}, h \neq l} G_{h l} X_{h}},
$$

$G_{l l}$ is the channel gain of link $l, G_{l h}$ the gain from the transmitter of link $l$ to the receiver of link $h$, and $N_{l}$ is the noise at the receiver of link $l$.

$K$-hop Interference: We assume a $K$-hop interference model where when node $i$ is transmitting, only links whose receivers are at most $K$-hop away from node $i$ will be interfered with. Denote by $\mathcal{I}_{l}$ the set of links whose transmitters are fewer than $K$ hops from $r(l)$, i.e.,

$$
\mathcal{I}_{l}=\{h:|t(h)-r(l)| \leq K, h \in \mathcal{E}\}
$$

The $K$-hop interference model is equivalent to the following statement:

$$
R_{l}(\mathbf{X})=R_{l}\left(\left\{X_{l}: l \in \mathcal{I}_{l}\right\}\right) \quad \forall l .
$$

For Gaussian channels, $K$-hop interference model implies that $G_{h l}=0$ if $|r(l)-t(h)|>K$.

Half Duplex: We assume half duplex model in this paper such that at each time:

(i) Each sender can only transmit to one receiver,

(ii) each receiver can only receive from one sender,

(iii) and, each node cannot transmit and receive simultaneously.

Note that the half-duplex constraint implies that no adjacent links can transmit simultaneously. Define $\mathcal{X}$ to be the set of $\mathbf{X}$ satisfying the half duplex constraint, i.e.,

$$
\mathcal{X}=\left\{\mathbf{X}: X_{l} X_{h}=0 \text { if } l, h \text { are adjacent }\right\} .
$$

Link Rate Region: We define the link rate region to be

$$
\Gamma:=\operatorname{conv}(\mathbf{R}(\mathbf{X})) \text {. }
$$

Traffic Flow: We consider a system with $\mathcal{F}$ flows, where each flow $[s, d]$ is associated with a source $s$ and destination $d$. Further, assume that time is discrete and let $\lambda_{[s, d]}(s, d \in$ $\mathcal{V})$ denote the packet generation rate of flow $[s, d]$. We assume that the flow arrival process is i.i.d., and define $\lambda=\left\{\lambda_{[s, d]}\right\}$. (We can easily extend the results to more general arrival processes.)

Throughput Region: A traffic vector $\boldsymbol{\lambda}$ is said to be supportable in network $\mathcal{G}$ if there exists a $\left\{\mu_{l}^{d}\right\}_{l \in \mathcal{E}}$ such that

(i) For a destination $d$ and node $n \neq d$,

$$
\sum_{s:[s, d] \in \mathcal{F}} \lambda_{[s, d]} 1_{\{n=s\}}+\sum_{\{l: r(l)=n\}} \mu_{l}^{d}=\sum_{\{l: t(l)=n\}} \mu_{l}^{d}
$$

(ii)

$$
\left\{\sum_{d \in \mathcal{V}} \mu_{l}^{d}\right\}_{l \in \mathcal{E}} \in \Gamma .
$$

We denote by $\Lambda_{\mathcal{G}}$ the set of supportable $\lambda$. (These are standard definitions - for more details and intuition we refer to $[14])$.

\section{Motivation AND Main Results}

Note that under the Gaussian channel model (1), the dependency between transmit power and link rates is nonconvex. A popular approach [5], [7] is to use the approximation

$$
R_{l}(\mathbf{X}) \approx \log \left(\operatorname{SINR}_{l}(\mathbf{X})\right)
$$

based on a high-SINR assumption, and then appropriately transform the variable to formulate a convex optimization problem. However, when the interference between links is strong, the receivers cannot be in the high-SINR regime at the same time. Then, the approximation under high-SINR could be quite inaccurate.

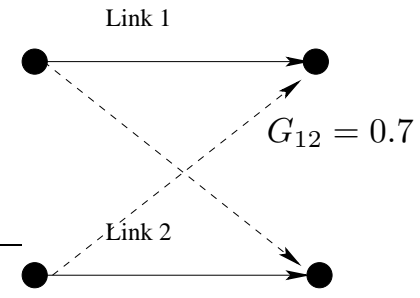

Fig. 2. A Two-Link Network

Consider a simple example with two links as in Figure 2 , where we assume that $\mathcal{P}=[0,10], G_{l l}=1, G_{12}=$ $G_{21}=0.7$, and $N_{l}=1$. Using a numerical calculation, the supportable rate-region $\left(R_{1}, R_{2}\right)$ is as depicted using the "+"-line in Figure 3. However, under the high-SINR assumption, the rate-region is as depicted using the " $*$ " line in Figure 3. We can see that the two rate-regions are quite different. More importantly, the power-control policies obtained with or without the high-SINR assumption will be different. For example, to maximize $R_{1}+R_{2}$, without the 
high-SINR assumption, the solution is $\left(X_{1}, X_{2}\right)=(10,0)$ or $\left(X_{1}, X_{2}\right)=(0,10)$. However, under the high-SINR assumption, $\left(X_{1}, X_{2}\right)=(5,5)$ becomes optimal, which however is the point minimizing $R_{1}+R_{2}$ in the original rate-region.

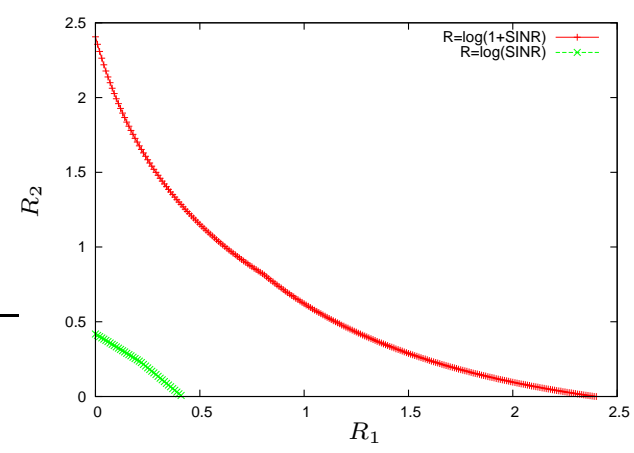

Fig. 3. Rate Region

Motivated by the observation above, we propose distributed power control algorithms without the high-SINR approximation in this paper. Our main results include the following:

(a) We develop a distributed power-control algorithm based on message passing, under which the power allocation $\mathbf{X}^{*}$ satisfies

$$
\mathbf{X}^{*}=\max _{\mathbf{X} \in \mathcal{X}}\left(\sum_{l \in \mathcal{E}} w_{l} R_{l}(\mathbf{X})\right) .
$$

for non-negative weights $w_{l}$.

(b) We describe a back-pressure-based routing/powercontrol algorithm, which stabilizes the network for any traffic $\boldsymbol{\lambda}$ such that $(1+\epsilon) \boldsymbol{\lambda} \in \Lambda_{\mathcal{G}}$.

(c) Assume that each flow has an utility function $U_{[s, d]}\left(\lambda_{[s, d]}\right)$ (see Section VI for more details). We describe a joint congestion-control and power-control algorithm, under which each flow has rate $\lambda_{[s, d]}^{*}$ such that

$$
\boldsymbol{\lambda}^{*}=\arg \max _{\boldsymbol{\lambda} \in \Lambda_{\mathcal{G}}} \sum_{[s, d] \in \mathcal{F}} U_{[s, d]}\left(\lambda_{[s, d]}\right) .
$$

Remark 1: Note that the network traffic is not considered in problem (a), so $\mathbf{X}^{*}$ is generally not the best power allocation from the network layer perspective. However, problem (a) is interesting because, to achieve throughput-optimality and utility maximization, we need to solve problem (a) with different weights $\left\{w_{l}, l \in \mathcal{E}\right\}$ at different time instances. Thus, the algorithm developed for problem (a) will be a key component in the algorithms for problems (b) and (c).

Remark 2: Due to page limitations, we only study linenetworks in this paper. However, our results can be extended to grid networks. Let us consider a grid network with $N$ nodes. The idea is to partition the grid into rectangles of (node) size $\sqrt{N} \times(K+1)$. By properly choosing some rectangles and turning off all nodes in them, the network can be divided into non-interference components. Then the scheduling/power allocation decision within each component can be obtained using the junction-tree algorithm described for the line-network. Obviously, we cannot always turning off the same rectangles, as this will block the traffic in these rectangles. Thus, the key challenge is to dynamically select rectangles to turn-off in order to achieve throughput optimality and utility maximization. With time complexity $O(V \sqrt{N}), 1 / V$-optimal (the solutions are $1 / V$ near to the optimal ones) algorithms can be developed for grid networks. Additional details are available in [15].

\section{Power Control via Distributed Message PASSING}

In this section, we first consider the following optimization problem:

$$
X^{*} \in \arg \max _{\mathbf{X} \in \mathcal{X}}\left(\sum_{l \in \mathcal{E}} w_{l} R_{l}(\mathbf{X})\right),
$$

where $\left\{w_{l}, l \in \mathcal{E}\right\}$ are non-negative weights associated with the links. Note that any solution to the above optimization problem results in an optimal power allocation and schedule. To solve the optimization problem above, we first rewrite the problem as a max-product problem. Given $\mathbf{X} \in \mathcal{X}$, we define the rate at a receiver $i \in \mathcal{V}$ as

$$
\tilde{R}_{i}(\mathbf{X})=\sum_{\{l: r(l)=i\}} w_{l} R_{l}(\mathbf{X}) .
$$

Therefore, the optimization problem (7) can be written as

$$
X^{*} \in \arg \max _{\mathbf{X} \in \mathcal{X}}\left(\sum_{i \in \mathcal{V}} \tilde{R}_{i}(\mathbf{X})\right) .
$$

Further define $f_{i}(\mathbf{X})=e^{\tilde{R}_{i}(\mathbf{X})}$, then the optimization problem (7) is equivalent to

$$
\begin{aligned}
X^{*} & \in \arg \max _{\mathbf{X} \in \mathcal{X}} \log \left(\prod_{i \in \mathcal{V}} f_{i}(\mathbf{X})\right), \\
& =\arg \max _{\mathbf{X} \in \mathcal{X}}\left(\prod_{i \in \mathcal{V}} f_{i}(\mathbf{X})\right),
\end{aligned}
$$

where

$$
f_{i}(\mathbf{X})=e^{\sum_{\{l: r(l)=i\}} w_{l} R_{l}(\mathbf{X})}
$$

For the $K$-hop interference model, we observe that $f_{i}(\mathbf{X})$ contains or depends only on a small number of decision variables $X_{l}$. Denote the set of links that determine the function $f_{i}($.$) by S_{i}$. Then we have

$$
S_{i}=\left\{\text { all links } \tilde{l}: \tilde{l} \in \cup_{l: r(l)=i} \mathcal{I}_{l}\right\} .
$$

Further, define the associated transmit power-levels by

$$
\mathbf{X}_{S_{i}}=\left\{X_{l}: l \in S_{i}\right\}
$$

(Note that $X_{l}=0$ corresponds to the link $l$ not being scheduled.) 
The optimization problem (7) thus reduces to

$$
X^{*} \in \arg \max _{\mathbf{X} \in \mathcal{X}} \prod_{i \in \mathcal{V}} f_{i}\left(\mathbf{X}_{S_{i}}\right)
$$

The power-control/scheduling problem has thus been recast as an optimization problem where the objective is to maximize a product function, which has been well studied in literature. This enables us to use techniques discussed in [11] to solve the power control problem.

Let $\mathbf{S}$ denote the set $\left\{S_{1}, S_{2}, \ldots, S_{n}\right\}$ where $S_{i}$ is defined in (10). Further, let $H_{i}: \mathcal{P}^{\left|S_{i}\right|} \rightarrow R$, be defined by

$$
H_{i}\left(\mathbf{X}_{S_{i}}\right)=\max _{\mathbf{X}_{S_{i}^{c}}} \prod_{i \in \mathcal{V}} f_{i}\left(\mathbf{X}_{S_{i}}\right) .
$$

where $S_{i}^{c}$ denotes the complement of the set $S_{i}$ with respect to $\mathcal{E}$.

Next, we describe the algorithm to find an optimal power allocating scheme using belief propagation techniques. The algorithm consists of two parts:

First, for each node $i \in \mathcal{V}$, we compute the function $H_{i}\left(\mathbf{X}_{S_{i}}\right)$ for each possible $\mathbf{X}_{S_{i}}$ using belief propagation (message passing) over a suitably constructed graph (a junction tree).

Second, for each node $i \in \mathcal{V}$, we use (12) to obtain the corresponding $\operatorname{set}^{1}\left\{\mathbf{X}_{S_{i}}^{*}\right\}$. These vectors correspond to possible choices of optimal transmit powers for interfering links with the receiver of node $i$ (i.e., the set of links that can be "heard" at receiver node $i$ ). We then demonstrate an algorithm that chooses a single power vector $\mathbf{X}_{S_{i}}^{*}$ at each node $i$ such that these vectors are consistent (i.e., each link's transmit power is the same value at all nodes).

\section{A. Part I: Junction Tree Algorithm}

The junction tree algorithm provides a means of computing (12) at each node $i$. The result of this computation will be used in the following section to determine the optimal transmit power levels and schedule.

Definition [11]: A junction tree is a tree with vertices indexed by $\left\{X_{S_{i}}\right\}$ where the subscript of vertex $X_{S_{i}}$ corresponds to a set $S_{i} \subset S$ satisfying the following property: For any two vertices $X_{S_{i}}$ and $X_{S_{j}}$, and any vertex $X_{S_{k}}$ on the path from $X_{S_{i}}$ to $X_{S_{j}}$, we have $S_{i} \bigcap S_{j} \subset S_{k}$.

Observe that the junction tree for line network with $K$-hop interference is a tree $T$ in which each vertex $X_{S_{i}}$ is connected to $X_{S_{i-1}}$ and $X_{S_{i+1}}$ except for the vertices $X_{S_{1}}$ and $X_{S_{N}}$ which are only connected to $X_{S_{2}}$ and $X_{S_{N-1}}$ respectively. In other words, the junction tree is a series (line) graph. Thus we can apply the standard max-product algorithm (junction tree algorithm) [11], [12] on this new series (line) graph.

The (synchronous) max-product algorithm exchanges messages (real-valued vectors) iteratively in order to determine $\left\{\mathbf{X}_{S_{i}}^{*}\right\}$ at each node $i$.

If the vertices $X_{S_{i}}$ and $X_{S_{j}}$ are connected (adjacent), then at each time-step $t$ the message passed from vertex $X_{S_{i}}$ to

\footnotetext{
${ }^{1}$ The outcome is a set because the optimization problem may not have an unique solution.
}

$X_{S_{j}}$ is a vector containing the values of the function $M_{i, j}^{t}$ : $\mathcal{P}^{\left|S_{i} \cap S_{j}\right|} \rightarrow R$, defined by

$M_{i, j}^{t}\left(\mathbf{X}_{S_{i} \cap S_{j}}\right)=\max _{\mathbf{X}_{S_{i} \backslash S_{j}}}\left\{f_{i}\left(\mathbf{X}_{S_{i}}\right) M_{k, i}^{t-1}\left(\mathbf{X}_{S_{k} \cap S_{i}}\right)\right\}$

where $\mathbf{X}_{S_{i} \cap S_{j}}=\left\{X_{l}: l \in S_{i} \cap S_{j}\right\}$.

The Junction Tree Algorithm [11]:

(i) At time-step 0 , each vertex $X_{S_{i}}$ sends the message vector 1 to its adjacent nodes. In other words, if $X_{S_{i}}$ and $X_{S_{j}}$ are adjacent, then vertex $X_{S_{i}}$ send the message $M_{i, j}^{0}\left(\mathbf{X}_{S_{i} \cap S_{j}}\right)=1$ to $X_{S_{j}}$.

(ii) At each successive time-step $t$, the vertex $X_{S_{i}}$ sends the message $M_{i, j}^{t}$ to its adjacent vertex $X_{S_{j}}$, where $M_{i, j}^{t}$ is defined in (13). Each vertex $X_{S_{i}}$ stops sending messages to its adjacent vertex $X_{S_{j}}$ when the node $X_{S_{i}}$ receives exactly the same message $M_{k, i}^{t}$ over two consecutive time-steps from its other adjacent vertex $X_{S_{k}}$. Once all nodes stop sending messages, we denote the terminal message sent from a vertex $\mathbf{X}_{S_{i}}$ to its adjacent vertex $\mathbf{X}_{S_{j}}$ by $M_{i, j}\left(\mathbf{X}_{S_{i} \cap S_{j}}\right)$.

(iii) Each vertex $\mathbf{X}_{S_{i}}$ computes $\tilde{H}_{i}\left(\mathbf{X}_{S_{i}}\right)$ defined as

$$
\begin{aligned}
& \tilde{H}_{i}\left(\mathbf{X}_{S_{i}}\right)= \\
& f_{i}\left(\mathbf{X}_{S_{i}}\right) \prod_{\{k: 1 \leq k \leq N, k=i \pm 1\}} M_{k, i}\left(\mathbf{X}_{S_{k} \cap S_{i}}\right) \text {. (14) }
\end{aligned}
$$

It is well known that the above algorithm converges to the correct marginals (or maximization in our case) in the case of tree networks [11], [12]. In other words, $(14)=(12)$.

Theorem 1: The junction tree algorithm for the line network with $K$-hop interference determines (12) in $N$ timesteps, where $N$ is the number of nodes in the network.

The above algorithm also naturally lends itself to an asynchronous implementation (see [12], [16] for additional details). As long as all messages and iterates are updated sufficiently large number of times ${ }^{2}$, we have the fixed points of the asynchronous algorithm coincide with the fixed points of the above described synchronous algorithm.

We next demonstrate a correspondence (map) between the message transmissions on the junction tree and actual (radio) transmissions over the line network.

The vertex $X_{S_{i}}$ is mapped to the node $i$. The function $f_{i}\left(\mathbf{X}_{S_{i}}\right)$ computation is done at node $i$. From (9), we have

$$
f_{i}\left(\mathbf{X}_{S_{i}}\right)=\prod_{\{l: r(l)=i\}}\left(1+\operatorname{SINR}_{l}\left(\mathbf{X}_{S_{i}}\right)\right)^{w_{l}}
$$

To ensure that the half-duplex constraints are satisfied, $f_{i}(\cdot)$ is set to 1 (or equivalently, data rate $=0$ ) for all the vectors of $\mathbf{X}_{S_{i}}$ which violate the half-duplex constraints.

The messages passed from the node $i$ to node $j$ correspond to the messages passed from vertex $\mathbf{X}_{S_{i}}$ to $\mathbf{X}_{S_{j}}$ in the junction tree $T$. The scheduling of these messages can be done in an asynchronous fashion as described before.

\footnotetext{
${ }^{2}$ The messages need to be exchanged a large enough number of times such the effect of each nodes' messages propagates across the entire network.
} 
The termination of this message passing algorithm results in each node $i$ having the entire description of the function $H_{i}\left(\mathbf{X}_{S_{i}}\right)$ over all possible values of the power-level vector $\mathbf{X}_{S_{i}}$ (defined in (11)).

B. Part II: Algorithm to determine an optimal transmit power schedule (i.e., $\mathbf{X}^{*}$ )

Each node searches over the $L^{4 k+2}$ length vector $\tilde{H}_{i}\left(\mathbf{X}_{S_{i}}\right)$ to find the optimal schedule that maximizes $\tilde{H}_{i}(\cdot)$. Formally,

$$
x_{S_{i}}^{*} \in \mathcal{X}_{S_{i}}^{*}=\arg \max \tilde{H}_{i}\left(\mathbf{X}_{S_{i}}\right)
$$

If the above search gives a unique value then node $i$ sets its power level defined by $\mathbf{X}_{S_{i}}^{*}$.

Otherwise, if there are multiple vectors of $\mathbf{X}_{S_{i}}^{*}$ that maximize $\tilde{H}_{i}(\cdot)$, then there exists multiple optimal power allocation schemes. We next describe a method to find a consistent optimal power allocation.

\section{Find the Optimal Power Allocation:}

(i) Node 1 picks an $x_{S_{1}}^{*}$ from the optimal solution set $\mathcal{X}_{S_{1}}^{*}$.

(ii) Node $i-1$ sends $x_{S_{i-1}}^{*}$ to node $i$. After node $i$ obtains $x_{S_{i-1}}^{*}$ from node $i-1$, it selects an $x_{S_{i}}^{*}$ from $\mathcal{X}_{S_{i}}^{*}$ such that $x_{S_{i-1}}^{*}[l]=x_{S_{i}}^{*}[l]$ for all $l \in S_{i-1} \cap S_{i}$, where $x_{S_{i}}^{*}[l]$ is the decision for link $l$ in vector $x_{S_{i}}$. (Note that node $i$ will only execute this step after it receives the message from node $i-1$.)

After $N$ steps, it is easy to see that the nodes will find an optimal and consistent $x_{S_{i}}^{*}$. Then, based on its (local) information $x_{l}^{*}(l: t(l)=i)$, node $i$ can transmit over each link $l$ at the appropriate power level.

The above implementation is not fully distributed in the sense that nodes sequentially pass messages (starting from node 1). The scheme can be easily made to asynchronously operate in a distributed manner by assigning a priority level of $i$ to node $i$ and partitioning the nodes into bins (groups of nodes), where each node is assigned to a single bin, and nodes within a bin are within radio range of each other (the bins are chosen a-priori and arbitrarily subject to the above property). Then, each node $i$ picks an optimal power level from $\mathcal{X}_{S_{i}}^{*}$ that is consistent with the power level selection of the highest priority node within its bin (the "locally" highest priority node initially picks any arbitrary optimal power level). After this initial power assignment, over successive iterations, each node $i$ picks an optimal power level from $\mathcal{X}_{S_{i}}^{*}$ that is consistent with the (previously chosen) power level selection of the highest priority node within its radio range. It is clear that after a sufficient number of iterations (linear in $N$ ), the above scheme converges to a globally optimal power allocation.

\section{Throughrut Optimality}

In Section IV, we developed a power control algorithm based on distributed message passing, which yields a power allocation $\mathbf{X}^{*}$ such that

$$
\mathbf{X}^{*} \in \arg \max _{\mathbf{X} \in \mathcal{X}}\left(\sum_{l \in \mathcal{E}} w_{l} R_{l}(\mathbf{X})\right) \text {. }
$$

While $\mathbf{X}^{*}$ maximizes the aggregated link rate, it might not be best power allocation from the network perspective. For example, if there is no flow over a certain link, there is no need to allocate any power to that link. Thus, the power levels should be adaptively chosen according to the network traffic.

In this section, we consider inelastic traffic, i.e., we assume $\boldsymbol{\lambda}$ is fixed. Assuming there exists $\epsilon>0$ such that $(1+\epsilon) \boldsymbol{\lambda} \epsilon$ $\Gamma$, we will develop a back-pressure-based power-control algorithm to stabilize the network without any knowledge of $\lambda$.

Now, assume that each node maintains a separate queue for every other node, and let $q_{d, n}[t]$ denote the length of queue maintained for node $d$ at node $n$. The adaptive power control algorithm, which is named as back-pressure-based power-control, is a combination of the messaging passing algorithm in Section IV and the back-pressure algorithm proposed in [8].

\section{Back-Pressure-Based Power-Control:}

(i) At time slot $t$, the transmitter of link $l, t(l)$, obtains

$$
\begin{aligned}
d_{t(l)}^{*}[t] & =\arg \max _{d>t(l)} q_{d, t(l)}^{\alpha}[t]-q_{d, r(l)}^{\alpha}[t] \\
w_{l}[t] & =\max _{d>t(l)}\left(q_{d, t(l)}^{\alpha}[t]-q_{d, r(l)}^{\alpha}\right)^{+}
\end{aligned}
$$

where $\alpha$ is a positive integer, and $(\cdot)^{+}=\max \{0, \cdot\}$.

(ii) Solve

$$
\mathbf{X}^{*}[t] \in \arg \max \sum_{l \in \mathcal{E}} w_{l}[t] R_{l}(\mathbf{X}),
$$

using message passing algorithms. Then transmitter $t(l)$ transmits packets in queue $d_{t(l)}^{*}[t]$ with power $X_{l}^{*}[t]$ to node $r(l)$.

Theorem 2: The back-pressure weighted power control algorithm is throughput optimal, i.e., all queues are bounded under the back-pressure weighted power control algorithm given $\lambda \in \Lambda_{\mathcal{G}}$.

Proof: The proof is similar to [8]. A complete proof can also be found in [15].

\section{UTILITY MAXIMIZATION}

In Section V, we considered inelastic traffic, and described a back-pressure based power control algorithm to support any traffic within the network throughput region. In this section, we study elastic traffic, where the traffic rates can be adaptively changed according to the network condition.

Corresponding to each flow is a strictly concave, twice differentiable utility function $U_{[s, d]}\left(\lambda_{[s, d]}\right)$, where $-1 / U_{[s, d]}^{\prime \prime}\left(\lambda_{[s, d]}\right)$ is positive and bounded for $\lambda_{[s, d]} \in(0, M]$ (see [17] for detailed conditions).

The objective in this section is to develop a distributed algorithm that maximizes the sum of utilities:

$$
\max _{\boldsymbol{\lambda} \in \Lambda} \sum_{[s, d] \in \mathcal{F}} U_{[s, d]}\left(\lambda_{[s, d]}\right) .
$$


The algorithm to achieve this combines the congestion controller described in [2], [17] along with the message passing power control scheme with a suitable choice of weights:

Congestion Controller: Flow $[s, d]$ transmits packets with rate $\lambda_{[s, d]}[t]$ into the queue maintained for destination $d$ at node $s$, where $\lambda_{[s, d]}[t]$ satisfies

$$
\lambda_{[s, d]}[t]=\min \left\{U_{[s, d]}^{\prime-1}\left(\frac{q_{d, s}[t]}{\kappa}\right), M\right\}
$$

Back-pressure Power Control: The network solves

$$
\mathbf{X}^{*}[t] \in \arg \max \sum_{l \in \mathcal{E}} w_{l}[t] R_{l}(\mathbf{X}),
$$

using the distributed message passing algorithm, where $w_{l}[t]$ is defined as in equation (16). Then transmitter $t(l)$ transmits packets in queue $d_{t(l)}^{*}[t]$ with power $X_{l}^{*}[t]$ to node $r(l)$.

Since $U_{[s, d]}$ is a strictly concave function, and $\Lambda$ is convex, the optimization problem (18) has an unique solution. We denote the unique solution by $\lambda^{*}$.

Theorem 3: Let $\lambda_{[s, d], \kappa}[t]$ denote the rate of flow $[s, d]$ transmitting at time $t$ under the joint congestion-control and power control algorithm with parameter $\kappa$, we have

$$
\lim _{\kappa \rightarrow \infty} \lim _{t \rightarrow \infty} E\left[\lambda_{[s, d], \kappa}[t]\right]=\lambda_{[s, d]}^{*} .
$$

Proof: The idea of the proof is similar to [2].

\section{Complete Physical Interference Model}

In this section, we study the optimality of our algorithms on a complete physical interference model, i.e., without the $K$-hop interference assumption. Now consider the system model for the line network as described in section II without the $K$-hop interference assumption. Furthermore, we assume that the adjacent nodes (single hop) $i$ and $j$ are separated by a distance $|i-j| \in\left[d_{\min }, d_{\max }\right]$, where $0<d_{\min }<d_{\max }<$ $\infty$. We also assume that the path loss coefficient between the transmitter of link $l$ and receiver of link $h$ is

$$
G_{h l}=\frac{C}{|t(l)-r(h)|^{\alpha}}
$$

where $C>0$ and $\alpha$ is the path loss exponent with $\alpha \geq 2$.

We first consider the optimization problem (7). Let $\mathbf{X}^{*}$ be the optimal power allocation for the complete physical interference model. if

We define a power allocation scheme $\mathbf{X}_{\epsilon}^{*}$ to be $\epsilon$-optimal

$$
\sum_{l \in \mathcal{E}} w_{l} R_{l}\left(\mathbf{X}^{*}\right)-\sum_{l \in \mathcal{E}} w_{l} R_{l}\left(\mathbf{X}_{\epsilon}^{*}\right) \leq \epsilon \sum_{l \in \mathcal{E}} w_{l} R_{l}\left(\mathbf{X}^{*}\right)
$$

In the following theorem, we show that for any $\epsilon>0$, there exists a $K(\epsilon)$ such that the algorithm proposed in Section IV yields an $\epsilon$-optimal power allocation scheme for the complete physical interference model.

Theorem 4: Given any $\epsilon>0$, choose

$$
K(\epsilon)=\frac{2 C^{2} P_{\max }^{2}}{\delta d_{\min }^{2}\left(d_{\min }^{2}+C P_{\max }\right)},
$$

where $\delta=1-e^{-c \epsilon}$, and $c=\log \left(1+\frac{C P_{\max }}{L d_{\max }^{2}\left(1+\frac{2 C P_{\max } \pi^{2}}{6 d_{\min }^{2}}\right)}\right)$. Then, the power allocation resulting from the message passing algorithm in Section IV applied to a $K(\epsilon)$-hop interference model approximation of the complete physical interference model is $\epsilon$-optimal.

Proof: A complete proof can also be found in [15].

The throughput optimality and utility maximization results in Sections V and VI can be easily extended for the complete physical interference model.

\section{REFERENCES}

[1] X.Lin and N. B. Shroff, "The impact of imperfect scheduling on crosslayer rate control in multihop wireless networks," in Proc. of the IEEE INFOCOM, Miami, Florida, March 2005.

[2] A. Eryilmaz and R. Srikant, "Fair resource allocation in wireless networks using queue-length-based scheduling and congestion control," in Proc. of the IEEE INFOCOM, Miami, Florida, March 2005.

[3] — , "Joint congestion control, routing and mac for stability and fairness in wireless networks," IEEE Journal on Selected Areas in Communications, vol. 24(8), pp. 1514-1524, Aug 2006.

[4] M. Johansson and L. Xiao, "Scheduling, routing and power allocation for fairness in wireless networks," in IEEE VTC-Spring, Milan, Italy, May 2004.

[5] M. Chiang, "Balancing transport and physical layers in wireless multihop networks: Jointly optimal congestion control and power control," IEEE Journal of Selected Areas in Communications, vol. 23(1), pp. 104-116, Jan 2005.

[6] D. Julian, M. Chiang, D. O’Neill, and S. Boyd, "Qos and fairness constrained convex optimization of resource allocation for wireless cellular and adhoc networks," in Proceedings of IEEE INFOCOM 2002, Jun 2002, pp. 477-486.

[7] Y. Xi and E. M. Yeh, "Node-based optimal distributed power control, routing, and congestion control in wireless networks," in Proc. of the Conference on Information Sciences and Systems, Princeton, NJ, Mar 2006.

[8] L. Tassiulas and A. Ephremides, "Stability properties of constrained queueing systems and scheduling policies for maximum throughput in multihop radio networks," IEEE Transactions on Automatic Control, vol. 37(12), pp. 1936-1948, Dec 1992.

[9] E. Modiano, D. Shah, and G. Zussman, "Maximizing throughput in wireless networks via gossip," in Proc. of ACM SIGMetric/Performance, Saint Malo, France, Jun 2006.

[10] L. Georgiadis, M. J. Neely, and L. Tassiulas, "Resource allocation and cross-layer control in wireless networks," Foundations and Trends in Networking, vol. 1, no. 1, pp. 1-144, 2006.

[11] S. M. Aji and R. J. McEliece, "The generalized distributed law," IEEE Transactions on Information Theory, vol. 46(2), pp. 325-343, Mar 2000.

[12] T. Richardson and R. Urbanke, Modern Coding Theory. Cambridge University Press, 2008.

[13] M. Bayati, B. Prabhakar, D. Shah, and M. Sharma, "Iterative scheduling algorithms," in Proceedings of IEEE Infocom, Anchorage, Alaska, USA, May 2007.

[14] L. Ying, R. Srikant, and D. Towsley, "Cluster-based back-pressure routing algorithm," in Proceedings of IEEE Infocom, Phoenix, AZ, 2008.

[15] A. Reddy, S. Shakkottai, and L. Ying, "distributed power control in wireless ad hoc networks using message passing: Throughput optimality and network utility maximization," in UT Austin Technical Report, 2008.

[16] C. Moallemi and B. V. Roy, "Convergence of the min-sum algorithm for convex optimization," in In Proceedings of the 45th Allerton Conference on Communication, Control and Computing, Monticello, IL, Sep 2007.

[17] A. Eryilmaz and R. Srikant, "Joint congestion control, routing and mac for stability and fairness in wireless networks," IEEE Journal on Selected Areas in Communications, special issue on Nonlinear Optimization of Communication Systems, vol. 24, no. 8, pp. 15141524, August 2006. 